\title{
Hantzsch-Like One-Pot Three-Component Synthesis of Heptaaza- dicyclopenta[a,j]anthracenes: A New Ring System
}

Amr M. Abdelmoniem

Farid M. Sroor

Muhammed A. Ramadan

Said A. S. Ghozlan

Ismail A. Abdelhamid

Synlett 2020, 31, 895.

The name of the author 'Ismail A. Abdelhamid' has been corrected for the final online and print version. 